\title{
Kinetic Sunyaev-Zel'dovich effect in rotating galaxy clusters from MUSIC simulations
}

\author{
Anna Silvia Baldi, ${ }^{1,2 \star}$ Marco De Petris, ${ }^{1}$ Federico Sembolini, ${ }^{1,3}$ \\ Gustavo Yepes, ${ }^{3,4}$ Weiguang Cui, ${ }^{3}$ Luca Lamagna ${ }^{1}$ \\ ${ }^{1}$ Dipartimento di Fisica, Sapienza Università di Roma, Piazzale Aldo Moro 5, I-00185 Roma, Italy \\ ${ }^{2}$ Dipartimento di Fisica, Università di Roma "Tor Vergata", Via della Ricerca Scientifica 1, I-00133 Roma, Italy \\ ${ }^{3}$ Departamento de Física Teórica, Módulo 8, Facultad de Ciencias, Universidad Autónoma de Madrid, E-28049 Madrid, Spain \\ ${ }^{4}$ Centro de Investigación Avanzada en Física Fundamental (CIAFF), Universidad Autónoma de Madrid, E-28049, Madrid, Spain
}

Accepted XXX. Received YYY; in original form ZZZ

\begin{abstract}
The masses of galaxy clusters are a key tool to constrain cosmology through the physics of large-scale structure formation and accretion. Mass estimates based on X-ray and Sunyaev-Zel'dovich measurements have been found to be affected by the contribution of non-thermal pressure components, due e.g. to kinetic gas energy. The characterization of possible ordered motions (e.g. rotation) of the intra-cluster medium could be important to recover cluster masses accurately. We update the study of gas rotation in clusters through the maps of the kinetic Sunyaev-Zel'dovich effect, using a large sample of massive synthetic galaxy clusters $\left(M_{\mathrm{vir}}>5 \times 10^{14} h^{-1} \mathrm{M}_{\odot}\right.$ at $\left.z=0\right)$ from MUSIC high-resolution simulations. We select few relaxed objects showing peculiar rotational features, as outlined in a companion work. To verify whether it is possible to reconstruct the expected radial profile of the rotational velocity, we fit the maps to a theoretical model accounting for a specific rotational law, referred as the vp $2 \mathrm{~b}$ model. We find that our procedure allows to recover the parameters describing the gas rotational velocity profile within two standard deviations, both with and without accounting for the bulk velocity of the cluster. The amplitude of the temperature distortion produced by the rotation is consistent with theoretical estimates found in the literature, and it is of the order of 23 per cent of the maximum signal produced by the cluster bulk motion. We also recover the bulk velocity projected on the line of sight consistently with the simulation true value.
\end{abstract}

Key words: methods: numerical - galaxies: clusters: general - cosmology: miscellaneous - cosmology: Sunyaev-Zel'dovich effect.

\section{INTRODUCTION}

The study of non-random motions within galaxy clusters is an important topic in modern astrophysics, especially to determine accurate estimates of their masses. Indeed, the most used methods to measure cluster masses are based on the simple assumption of hydrostatic equilibrium (see for instance Voit 2005), that accounts for the pressure contribution due to random thermal motions only. Results from numerical simulations of galaxy clusters show that hydrostatic masses underestimate the real values by fractions of the order of 10-20 per cent (see e.g. Rasia et al. 2006; Meneghetti et al. 2010; Nelson et al. 2012; Biffi et al. 2016). One of the possible explanations for this discrepancy is that it is

* E-mail: annasilvia.baldi@uniroma1.it necessary to account for additional pressure support coming both from turbulent motions (Rasia, Tormen \& Moscardini 2004; Lau, Kravtsov \& Nagai 2009), and from coherent streams or rotation (as investigated by e.g. Fang, Humphrey \& Buote 2009; Biffi, Dolag \& Böhringer 2011; Biffi, Dolag \& Böhringer 2013). In the recent work by Eckert et al. (2018), a quantitative estimate of the non-thermal pressure support in the ICM is addressed, using for the first time observational $\mathrm{X}$-ray and microwave data for a small sample of nearby massive clusters. The authors find that the median value of the non-thermal vs thermal pressure ratio is of the order of 10 per cent at the virial radius, which is smaller than the predictions from numerical simulations. Nevertheless, they also report an exception for the Abell cluster A2319, for which this ratio is $\sim 50$ per cent instead.

Ordered motions - particularly rotation - are, never- 
theless, very challenging to measure. As reviewed in Hamden et al. (2010), several approaches for determining the presence of cluster rotation exist. Indeed, it can be investigated from spectroscopic measurements towards the galaxy members at optical wavelengths (Hwang \& Lee 2007; Tovmassian 2015; Manolopoulou \& Plionis 2017), or from observations of the diffuse intra-cluster medium (ICM hereafter) at X-ray (Bianconi, Ettori \& Nipoti 2013; Liu et al. 2015) and microwave wavelengths (Cooray \& Chen 2002; Chluba \& Mannheim 2002; Sunyaev, Norman \& Bryan 2003). The latter, in particular, are possible through the Sunyaev-Zel'dovich (SZ) effect, that is produced by the inverse Compton scattering of the hot free electrons in the ICM and the photons of the cosmic microwave background (CMB) (Sunyaev \& Zel'dovich 1970, 1980). The SZ effect is observed as a variation in the temperature (or brightness) of the CMB spectrum, and can be separated in two components, namely the thermal and the kinetic SZ effect (tSZ and kSZ, respectively; see e.g. Rephaeli 1995, for a review). The tSZ is produced by the random thermal motion of the electrons, while the kSZ effect is attributed to the motion of the gas in the cluster as a whole with respect to the CMB reference frame. Quantitatively, the $\mathrm{kSZ}$ signal is proportional to the integral of the electron number density along the line of sight, times the projection of the cluster velocity on the same line of sight. This implies that, in principle, it would be possible to derive the kinetic properties of the ICM from observations of the $\mathrm{kSZ}$, as discussed e.g. in Dupke \& Bregman (2002). Some attempts to constrain cluster velocities with the kSZ have been reported in several works through the years using data from simulations (e.g. in Nagai, Kravtsov \& Kosowsky 2003), as well as from observations with different instruments with increasing sensitivity and angular resolution (see e.g. Holzapfel et al. 1997; Benson et al. 2003; Kashlinsky et al. 2008; Sayers et al. 2013; Sayers et al. 2016). For instance, a very recent example is the work by Adam et al. (2017), where they report the first high-significance detection of the $\mathrm{kSZ}$ towards two cluster substructures in MACS J0717.5+3745 (with 3.4 and 5.1 $\sigma$ significance, respectively), from measurements at $260 \mathrm{GHz}$ with an effective angular resolution of 22 arcseconds. Also, cluster motions have been detected with high significance in a statistical fashion, by estimating the pairwise momentum between couples of galaxy clusters using CMB data (Hand et al. 2012; Planck Collaboration et al. 2016; Li et al. 2018). It is worth to stress that, to quantify cluster velocities from single-cluster measurements, it is necessary to use complementary data from X-ray observations, since the kSZ alone is not sufficient to disentangle the contribution to the total signal coming from both the electron density - that can be inferred from measurements of the X-ray luminosity - and the velocity. Moreover, X-ray spectroscopy can also be an independent observational probe for the study of ICM motions. For instance, recent observations of the Perseus cluster with the Hitomi satellite, allowed to establish with high significance the presence of motions of the gas from measurements of the shift of metal spectral lines with unprecedented resolution (Hitomi Collaboration et al. 2016, 2018).

The possibility of exploiting the kSZ effect to detect rotational motions in clusters has been discussed in Cooray \& Chen (2002) (CC02, hereafter) for the first time. Assuming a simple solid body rotation for the gas, they find that the kSZ temperature anisotropy induced by this motion would be characterized by a dipolar pattern, produced by the projection on the line of sight of the velocity of the receding and approaching gas with respect to the observer. In Chluba \& Mannheim (2002), assuming the results of CC02 as a starting basis, they report a detailed analytical development of the characteristics of this signal as a function of cluster physical parameters, and they give some estimates of the expected amplitude, as measured with interferometric methods towards a set of candidates from nearby clusters. In both the aforementioned works, the amplitude of the signal at the dipole peak is estimated to range between few $\mu \mathrm{K}$ and tens of $\mu \mathrm{K}$, depending on the orientation of the line of sight with respect to the rotation axis and on the dynamical state of the observed cluster. Indeed, it is possible to explain the presence of a bulk rotation in galaxy clusters with the occurrence of a recent merging event (see e.g. Ricker \& Sarazin 2001), whose fetaures can be also inferred from the $\mathrm{kSZ}$ maps themselves, as shown in the recent work of Zhang, Yu \& Lu (2018).

At the time of writing no application of the $\mathrm{kSZ}$ for the study of cluster rotation has been reported so far. In fact, this is a very challenging task which, observationally speaking, is primarily limited by the resolution reached by current operating instruments, as well as from high relative errors of the inferred cluster velocity due to instrumental and astrophysical contaminants, or to uncertainties in the reconstruction of complementary ICM properties (e.g. the temperature). Towards a possible future joint multi-wavelength detection of rotational motions in real clusters, modern highresolution gas-dynamical simulations are extremely valuable tools for preliminary analyses. A very first test on the detectability of turbulent and ordered motions through the $\mathrm{kSZ}$ is reported in Sunyaev et al. (2003), where the authors consider a large, isolated galaxy cluster from a cosmological simulation populated with dark matter and non-radiative gas physics. Its $\mathrm{kSZ}$ map shows the presence of a possible coherent rotation, that can be deduced from two clearly distinguishable spots of opposite sign in the innermost regions of the cluster. In the present work, we take a step forward, making use of more realistic high-resolution cluster simulations to improve the estimate of the rotational $\mathrm{kSZ}$ signal, and to possibly describe the ICM rotation with a more suitable model for the rotational velocity, different from the simple solid body. In particular, we study for the first time the temperature distortion produced by the $\mathrm{kSZ}$ effect due to the ICM rotation, in massive galaxy clusters extracted from MUSIC simulations (Sembolini et al. 2013). We select a small sample of relaxed clusters showing also rotational features, as outlined in a complementary work focused on the properties of the angular momentum and tangential velocity of both ICM and dark matter in the same data set (Baldi et al. 2017, B17 hereafter). By applying B17's vp2b model to describe the radial profile of the tangential velocity, we test whether the expected rotational properties can be recovered from a fit to the $\mathrm{kSZ}$ maps, both with and without accounting for the overall cluster bulk motion. Since this preliminary analysis is not linked to any particular experiment, in this work we use clean data without accounting for noise or contaminations of astrophysical and instrumental origin. The full treatment of these effects will be included in a forthcoming work. 
This paper is organized as follows. In section 2 we present the cluster data set used for this study. In section 3 we describe the theoretical and the synthetic kSZ maps, whose analysis is reported and discussed in section 4 . We eventually summarize our conclusions in section 5 .

\section{DATA SET}

The simulated galaxy clusters analysed in this work constitute a small sub-sample of objects extracted from the MU$\mathrm{SIC}^{1}$ hydrodynamical $N$-body simulation project (see Sembolini et al. 2013, for details). MUSIC simulations have been run with the GADGET-3 TreePM+SPH code (Springel 2005). The initial conditions were extracted from two different parent simulations: MareNostrum Universe (Gottlöber \& Yepes 2007), constituting the MUSIC-1 sub-set, and MultiDark (Prada et al. 2012), constituting the MUSIC-2 sub-set. All the clusters were simulated using two different models to describe the baryonic component: one including only smoothed particle hydrodynamics (SPH) and gravity forces (NR subset), and one adding multi-phase inter-stellar medium modelling and radiative physics processes including radiative cooling, UV photoionization, star formation and supernova feedback (CSF subset), see Sembolini et al. (2013) for further details. Here we focus on the 258 most massive objects from MUSIC-2, having virial mass $M_{\text {vir }}>5 \times 10^{14} h^{-1} \mathrm{M}_{\odot}$ at redshift $z=0$, that have been already analysed in previous works to study SZ and X-ray scaling relations (Sembolini et al. 2013; Biffi et al. 2014; Sembolini et al. 2014), rotational features (B17) and SZ-derived morphological properties (Cialone et al. 2018).

To avoid possible contamination from the motion of large substructures in the kSZ maps, we limit our analysis to a small sub-sample of objects which feature $(i)$ a relaxed dynamical state, and $(i i)$ a sufficiently large spin parameter of the gas. Following the same procedure of B17, we first determine the dynamical state using two indicators derived from the simulation. The first one is the offset between the position of the centre of mass of the cluster and the position of the density peak, normalized to the virial radius, $\Delta r$. The second one is the ratio between the mass of the largest sub-structure in the cluster and the virial mass of the cluster itself, $M_{\text {sub }} / M_{\text {vir }}$. The detailed relation between these two indicators and their connection with the estimator of the cluster dynamical equilibrium can be found in Cui et al. (2017). We select the clusters that fulfil both conditions $\Delta r<0.1$ and $M_{\mathrm{sub}} / M_{\mathrm{vir}}<0.1$. After selecting these relaxed clusters, we apply a further selection based on the values of the spin parameter of the gas, $\lambda_{\text {gas }}$, considering only those having $\lambda_{\text {gas }}>0.07$, as we did in B17. With both these selection criteria, our final sample is reduced to six objects with possibly detectable rotation, whose main properties are listed in Table 1. The different modelling used to describe the baryon physics in MUSIC simulations (e.g. cooling and star formation) has been found to be not relevant for the gas rotational properties (see B17), which is in agreement with the results of Cui et al. (2016). For this reason, we limit here our analysis to the data from the non-radiative run, that does

1 http://music.ft.uam.es
Table 1. Identifier, virial mass, virial radius and spin parameter of the gas of the six relaxed and rotating MUSIC clusters analysed in this work (see text).

\begin{tabular}{cccc}
\hline cluster ID & $M_{\text {vir }}\left(\times 10^{15} \mathrm{M}_{\odot}\right)$ & $R_{\text {vir }}(\mathrm{kpc})$ & $\lambda_{\text {gas }}$ \\
\hline 46 & 1.17 & 2756 & 0.0785 \\
93 & 1.90 & 3241 & 0.0769 \\
98 & 1.61 & 3071 & 0.0735 \\
103 & 1.02 & 2633 & 0.0746 \\
205 & 1.24 & 2813 & 0.0763 \\
256 & 1.31 & 2867 & 0.0714 \\
\hline
\end{tabular}

not account for radiative gas physics. In these simulations the gas mass particle is set to $m_{\text {gas }}=1.9 \times 10^{8} h^{-1} \mathrm{M}_{\odot}$, while dark matter particles have mass $m_{\mathrm{DM}}=9.0 \times 10^{8} h^{-1} \mathrm{M}_{\odot}$.

We assume throughout the paper the same cosmological model adopted in MUSIC-2 and in the MultiDark parent simulation, which takes the parameter values from the best fit to the $W M A P 7+\mathrm{BAO}+\mathrm{SNI}$ data: $\Omega_{m}=0.27$, $\Omega_{b}=0.0469, \Omega_{\Lambda}=0.73, \sigma_{8}=0.82, n=0.95$ and $h=0.7$ (Komatsu et al. 2011).

\section{METHODS}

This section illustrates the theoretical model used to describe the rotation of the ICM in our clusters, and the method we use to compute the kSZ maps from the available data.

\subsection{Theoretical kSZ maps}

In the non-relativistic regime (Kompaneets 1957) the temperature shift produced by the kSZ observed along the direction identified by the vector $\hat{n}$, can be written as (e.g. Rephaeli 1995):

$\frac{\Delta T_{\mathrm{kSZ}}(\hat{n})}{T_{\mathrm{CMB}}}=-\frac{\sigma_{T}}{c} \int_{\mathrm{los}} n_{e} v_{p} d l$,

where $T_{\mathrm{CMB}}$ is the $\mathrm{CMB}$ monopole temperature, $T_{\mathrm{CMB}}=$ $(2.725 \pm 0.001) \mathrm{K}$ (Mather et al. 1999; Fixsen 2009) , $\sigma_{T}$ is Thomson cross section, $c$ is the speed of light, $n_{e}$ is the electron number density, and $v_{p}$ is the projection of the gas velocity on the line of sight (los).

If the gas rotates according to a law described by a generic angular velocity $\omega(r)$ along the cluster radius, equation (1) for such rotational component becomes:

$\frac{\Delta T_{\mathrm{kSZ}, \mathrm{r}}(R, \phi)}{T_{\mathrm{CMB}}}=-\frac{\sigma_{T}}{c} R \cos \phi \sin i \int_{R}^{R_{\mathrm{vir}}} n_{e}(r) \omega(r) \frac{2 r d r}{\sqrt{r^{2}-R^{2}}}$

as prescribed in CC02. $R$ and $\phi$ in equation (2) are the polar coordinates of the map in the plane of the sky, while $r$ is the three-dimensional radial distance from the cluster centre of mass. The $\sin i$ factor accounts for the orientation, given by the angle $i$, of the line of sight with respect to the rotation axis of the gas. If the line of sight is orthogonal or parallel with respect to the rotation axis, the amplitude of the signal will be therefore maximum or null, respectively. 


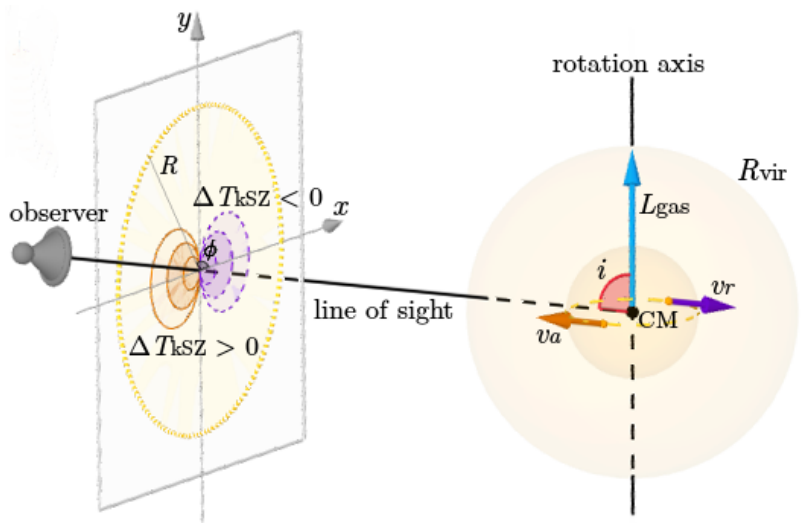

Figure 1. Sketch of the expected kSZ map from a rotating cluster, assuming the best observational configuration (i.e. with the line of sight orthogonal to the axis of rotation). The gas distribution in the cluster is assumed to be spherically symmetric, with the rotation axis aligned with the angular momentum vector of the gas as measured at the virial radius $\left(\boldsymbol{L}_{\text {gas }}\right)$. The velocity vectors, $\boldsymbol{v}_{a}$ and $\boldsymbol{v}_{r}$, indicate respectively the approaching and receding velocity components along the line of sight for two generic gas particles, located at the same radial distance from the cluster centre of mass.

A simple sketch illustrating the best observational configuration (with $i=90^{\circ}$ ) is represented in Fig. 1, in which the expected dipole-shaped pattern can be seen in the map as two symmetric spots - a positive one for approaching gas and a negative one for receding gas - aligned horizontally with respect to the projected axis of rotation.

In the most general case, the kSZ signal from a cluster is not only due to a pure rotational motion. Indeed, a contribution from the cluster bulk velocity is also present, and it may be dominant with respect to the rotation, depending on the projection of this velocity on the line of sight. The full model describing the theoretical $\mathrm{kSZ}$ due to a possible rotation plus the bulk motion is therefore:

$$
\begin{aligned}
\frac{\Delta T_{\mathrm{kSZ}}(R, \phi)}{T_{\mathrm{CMB}}} & =\frac{\Delta T_{\mathrm{kSZ}, \mathrm{r}}(R, \phi)}{T_{\mathrm{CMB}}}+ \\
& -\frac{\sigma_{T}}{c} v_{\mathrm{bulk}} \int_{R}^{R_{\mathrm{vir}}} n_{e}(r) \frac{2 r d r}{\sqrt{r^{2}-R^{2}}} .
\end{aligned}
$$

We denote with $v_{\text {bulk }}$ the projection of the cluster bulk velocity of the gas on the line of sight; the $\Delta T_{\mathrm{kSZ}, \mathrm{r}}(R, \phi) / T_{\mathrm{CMB}}$ term for the rotational component is given in equation (2). In this case, the expected signal is characterized by an asymmetric dipolar pattern, depending on the dominating approaching or receding $v_{\text {bulk }}$ at a given line of sight.

As can be seen from equations (2) and (3), the calculation of the theoretical $\mathrm{kSZ}$ maps requires to compute the integral over the line of sight of the electron number density profile, $n_{e}(r)$, and of the angular velocity profile, $\omega(r)$. The two analytical expressions we adopt to describe these profiles are detailed as follows.

- Electron number density: in real-life observations of clusters, the SZ effect alone cannot be used to constrain all the thermodynamic and dynamical properties of the ICM, as pointed out also in section 1 . Thus, in order to derive the parameters characterizing the rotational velocity it is nec-

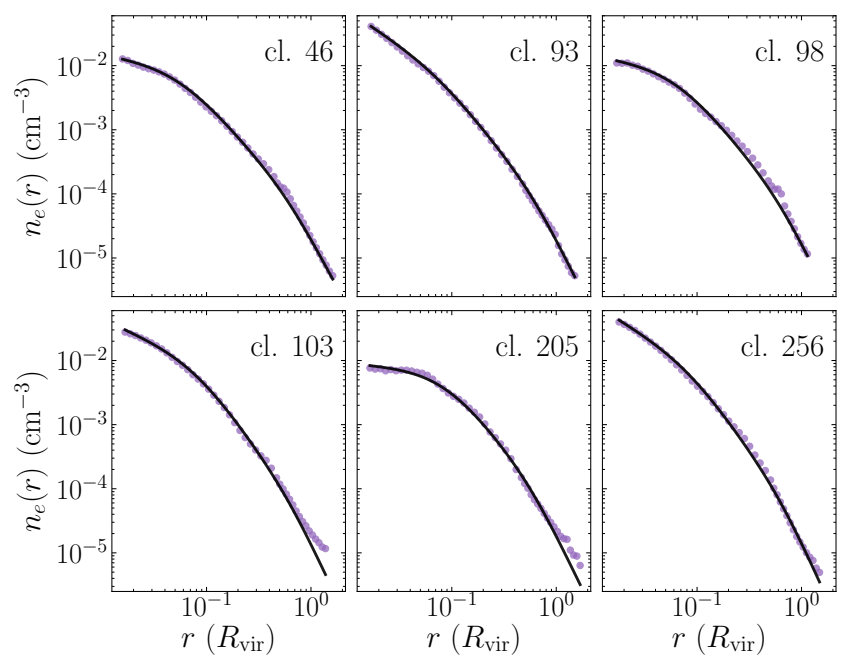

Figure 2. Radial profiles of the electron number density of the clusters in our sample. Purple dots are the median values computed according to equation (4); solid black lines represent the best-fit curves described by the simplified Vikhlinin model of equation (5) with the parameters listed in Table 2.

essary to have an independent measurement of the electron number density, and a possible estimate of the analytical model describing its radial profile, $n_{e}(r)$. This information has to be provided by ancillary X-ray observations, that allow to recover cluster densities at radii up to the virial radius (see e.g. Tchernin et al. 2016, and references therein). In our case, instead of using mock X-ray data, we make use of the numerical profiles derived from the cluster data provided by the simulation, and we fit them to a suitable theoretical model. The numerical profiles are computed as described in Sembolini et al. (2013), following:

$n_{e}(r)=N_{e}(r) \rho_{\mathrm{gas}}(r) \frac{Y_{H}}{m_{p}}$,

where $N_{e}(r)$ and $\rho_{\text {gas }}(r)$ are the number of electrons and the gas density at the cluster radius $r, Y_{H}=0.76$ is the hydrogen abundance referred to the gas particle, and $m_{p}$ is the proton mass. The model we use to describe $n_{e}(r)$ is a simplified six-parameter version of the equation proposed in Vikhlinin et al. (2006), that is:

$n_{e}(r)=n_{0} \frac{\left(r / r_{c}\right)^{-\frac{\alpha}{2}}}{\left[1+\left(r / r_{c}\right)^{2}\right]^{\frac{3 \beta}{2}-\frac{\alpha}{4}}} \frac{1}{\left[1+\left(r / r_{s}\right)^{\gamma}\right]^{\frac{\varepsilon}{2 \gamma}}}$,

where $n_{0}$ is the central density, $r_{c}$ and $r_{s}$ are scale radii, and $\alpha, \beta$ and $\varepsilon$ control the slopes of the profile at different radii. To determine the best-fit values of the free parameters $\left(n_{0}, r_{c}, \alpha, \beta, r_{s}\right.$ and $\left.\varepsilon\right)$, we use a Markov chain Monte Carlo procedure. The slopes $\alpha, \beta$ and $\varepsilon$, are all constrained to be positive, with the additional condition $\varepsilon<5$; the $\gamma$ parameter instead is kept fixed to 3.0 (Vikhlinin et al. 2006). We summarize the results in Fig. 2, showing the data and the best-fit curves, and in Table 2, where we list the parameter values.

- Angular velocity: the complementary work of B17 shows that the possible rotation of the gas in our cluster sample can be described by a generalized radial profile of the tangential velocity, rather than by a solid body model (differently from 
Table 2. Parameter values from the fit of radial profiles of the electron number density derived from the simulation to the simplified Vikhlinin model of equation (5). The $\gamma$ parameter has been kept fixed to 3.0 for all the clusters.

\begin{tabular}{ccccccc}
\hline cluster ID & $n_{0}\left(\mathrm{~cm}^{-3}\right)$ & $r_{c}\left(R_{\mathrm{vir}}\right)$ & $\alpha$ & $\beta$ & $r_{s}\left(R_{\mathrm{vir}}\right)$ & $\varepsilon$ \\
\hline 46 & $0.019 \pm 0.004$ & $0.056 \pm 0.004$ & $0.6 \pm 0.3$ & $0.63 \pm 0.08$ & $0.59 \pm 0.08$ & $2.3 \pm 1.2$ \\
93 & $0.019 \pm 0.005$ & $0.070 \pm 0.005$ & $2.1 \pm 0.2$ & $0.69 \pm 0.07$ & $0.61 \pm 0.07$ & $2.5 \pm 1.3$ \\
98 & $0.018 \pm 0.004$ & $0.064 \pm 0.004$ & $0.5 \pm 0.3$ & $0.7 \pm 0.1$ & $0.6 \pm 0.1$ & $2.6 \pm 1.5$ \\
103 & $0.021 \pm 0.004$ & $0.078 \pm 0.004$ & $1.4 \pm 0.2$ & $0.79 \pm 0.09$ & $0.63 \pm 0.09$ & $2.3 \pm 1.4$ \\
205 & $0.014 \pm 0.002$ & $0.088 \pm 0.002$ & $0.2 \pm 0.2$ & $0.7 \pm 0.1$ & $0.6 \pm 0.1$ & $2.5 \pm 1.4$ \\
256 & $0.020 \pm 0.005$ & $0.082 \pm 0.005$ & $1.9 \pm 0.2$ & $0.79 \pm 0.08$ & $0.64 \pm 0.08$ & $2.6 \pm 1.4$ \\
\hline
\end{tabular}

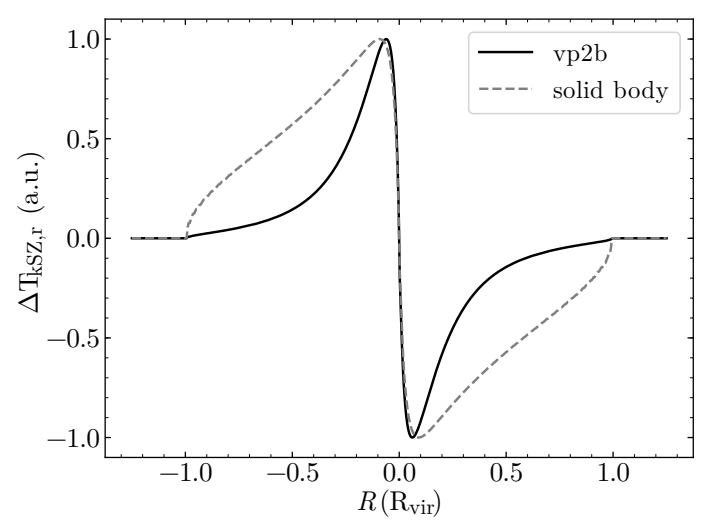

Figure 3. Central cuts along the theoretical maps of the rotational kSZ effect, computed using the vp2b and a solid body model to derive the angular velocity profile in equation (2). The two maps have been normalized, in order to highlight the differences in the shape of the cuts along the dipole.

CC02). We call this law vp2b model following the notation of B17, and we derive the corresponding radial profile of the angular velocity as:

$\omega(r)=\frac{\operatorname{vp} 2 \mathrm{~b}(r)}{r}=\frac{v_{t 0} / r_{0}}{1+\left(r / r_{0}\right)^{2}}$,

being $r_{0}$ and $v_{t 0}$ the parameters of the vp $2 \mathrm{~b}$ model, i.e. the scale radius and half of the velocity at this radius, $v_{t 0}=$ $\operatorname{vp} 2 \mathrm{~b}\left(r_{0}\right) / 2$ (see also equation (10) of B17).

Fig. 3 shows the central cuts along an example theoretical $\mathrm{kSZ}$ map computed according to equation (2), using the solid body model - with constant angular velocity $\omega-$ and the vp2b model, for a fixed profile of the electron number density. These cuts show that the dipole spots, which have here the same amplitude for comparison purposes, are more broadened in the case of constant angular velocity. The spatial scale of the dipole, that can be estimated as the distance between the maximum and the minimum peaks, is of the order of $\approx 0.2 R_{\mathrm{vir}}$ for both models. This is because this separation has a stronger dependence on the parameters setting the slopes of the electron number density profile - which has been kept fixed here - rather than on the velocity profile, in agreement with the results from Chluba \& Mannheim (2002).

\subsection{Simulated kSZ maps}

The kSZ maps of the synthetic clusters in our data set have been produced using the pymsz ${ }^{2}$ package, which provides mock observations of both the thermal and kinetic SZ effect. The kSZ temperature signal is computed as:

$$
\frac{\Delta T_{\mathrm{kSZ}}(\hat{n})}{T_{\mathrm{CMB}}}=-\frac{\sigma_{T}}{c D_{A}^{2}} \sum_{i}^{N_{P}} N_{e, i} v_{p, i} W_{p}\left(r_{i}, h_{s}\right),
$$

where $D_{A}$ is the angular diameter distance of the cluster. The sum extends over the total number of particles along the line of sight, $N_{P}$, each being located at a distance $r_{i}$ from the centre of mass, having projected velocity $v_{p, i}$ and containing $N_{e, i}$ electrons. The $W_{p}$ function is the SPH smoothing kernel of the simulation (see Sembolini et al. 2013). It is used to smear the $\mathrm{kSZ}$ from each gas particle to the projected image pixels, being $h_{s}$ the smoothing length of the gas particles. We compute the maps as described in equation (7) in two different versions, to match the only rotational and the rotational+bulk cases (corresponding to the theoretical prescriptions of equations (2) and (3), respectively). To separate the rotational component, we simply subtract the cluster bulk velocity, estimated as the average gas particle velocity, from the velocity of all single gas particles. In this way we can fit separately the kSZ maps computed from the data to the corresponding model in the two configurations, in order to establish whether it is possible to recover the expected rotational properties. Clearly, this is a simplification that cannot be used when dealing with real data, since it is not possible to separate the bulk component from the total signal, though some complementary methods to estimate the peculiar velocity could be used (e.g. the Tully-Fisher relation in the case of nearby clusters, see Kashlinsky et al. 2009).

With the aim to maximise the rotational signal, we choose the best observational configuration to detect the dipole, that corresponds to keep the angle between the line of sight and the rotation axis fixed to $i=90^{\circ}$ (edge-on with respect to the rotation axis). To get the corresponding projection, we perform a change of coordinate system, by transforming all the particle positions and velocities according to a suitable rotation matrix. Since we assume, as zero-th order approximation, that the rotation axis coincides with the direction of the angular momentum vector of the gas computed at the virial radius, we construct the rotation matrix so that the $z$ axis of the rotated reference frame coincides with this vector. With this choice the $x$ axis of the map should be aligned with the dipole spots, while the $y$ axis

2 https://github.com/weiguangcui/pymsz 
should correspond to the projection of the rotation axis on the plane of the sky, as also illustrated in Fig. 1. We want to stress here that we adopt these simplifications just to investigate the detectability of the rotation in suitable candidate clusters (relaxed with large spin parameter) using the kSZ at the best observational configuration.

In order to validate the effective rotational origin of the dipole pattern, we produce different projections for each cluster, obtained by selecting different lines of sight, all lying on the orthogonal plane to the rotation axis, always fulfilling the edge-on condition. Each line of sight is identified by the angle $\theta_{\text {los }}$, that indicates the separation with respect to the reference line of sight, having $\theta_{\text {los }}=0^{\circ}$. We take a total of six lines of sight, separated by uniform steps of $\Delta \theta_{\text {los }}=30^{\circ}$, so that $0^{\circ} \leq \theta_{\text {los }} \leq 150^{\circ}$. If a dipole is present because of ICM rotation, its approaching and receding spots should show the same sign and orientation in the maps, regardless of the particular line of sight chosen for the projection. As the subtraction of the cluster bulk velocity, this simplification in the interpretation of the results is also possible only when dealing with data from simulations, since observations can be made along only one line of sight.

The maps of each cluster extend over $2.5 R_{\mathrm{vir}}$ on a side, with a pixel size $d_{\text {pix }}=5 \times 10^{-3} R_{\text {vir }}$ that is of the order of $\approx 10 \mathrm{kpc}$. For practical reasons, we assume the analysed clusters to be located at $z=0.05$, instead of $z=0$. With this choice, according to the cosmological model adopted in the simulation, the angular diameter distance is $200.7 \mathrm{Mpc}$. The angular size of each pixel is therefore of the order of 10 arcsec, and the maps span 2.4 degrees on each side. To get results that can be useful for possible future applications to data from real experiments, we reduce the angular resolution of our simulated maps. To this end, we apply a smoothing with a Gaussian filter having full width at half maximum equal to 20 arcsec, which is compatible with the resolution of currently operating microwave instruments (e.g. NIKA2 at $\sim 200 \mathrm{GHz}$ ).

Fig. 4 shows the kSZ maps for cluster 93 - which is the most massive cluster in the sample - smoothed at 20 arcsec, for different lines of sight as described above. Top panels show the maps generated after the subtraction of the cluster bulk velocity which, on the contrary, is included in the maps shown in the bottom panels. It can be seen that, in general, all maps for cluster 93 reported in Fig. 4 show a dipole-like feature with horizontal alignment, and with spots having the same sign at all different projections. These characteristics confirm that cluster 93 is a good candidate for the inspection of a possible rotation of the ICM through the kSZ maps. In the maps in the right panels in Fig. 4 it is possible to see how the dipole gets distorted because of the dominating approaching component of the bulk velocity with respect to the observer for lines of sight having $\theta_{\text {los }} \leq 90^{\circ}$. The cluster bulk velocity projection is almost null for $\theta_{\text {los }} \geq 120^{\circ}$ instead, so that the rotational signal remains practically unaffected. A number of small-scale signal features can be identified in all the maps in Fig. 4, because of the presence of sub-structures. According to the relaxation criteria that we imposed to select the clusters in our sample (see section 2 for details), the masses of these sub-structures are smaller than ten per cent of the mass of the main halo. They may produce, in some cases, significant outliers in the kSZ temperature maps due to their high velocity projected on the line of sight. Despite

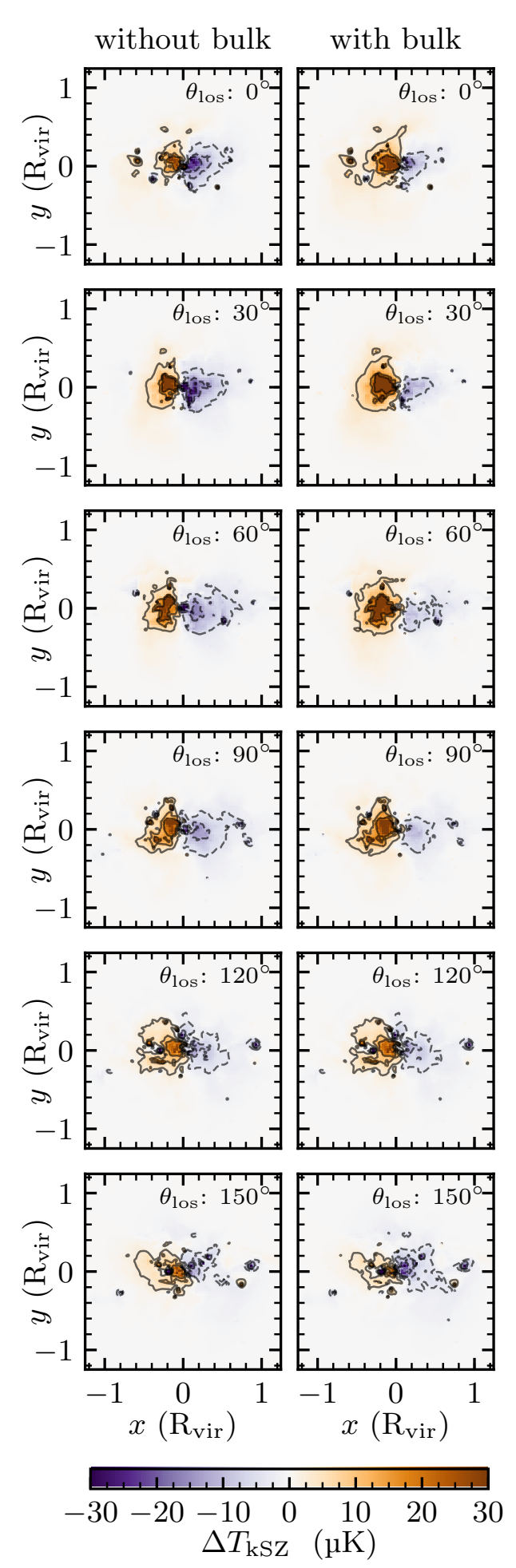

Figure 4. Maps of the temperature shift produced by the kSZ effect for cluster 93, obtained from different projections as described in the text, and smoothed at 20 arcsec. Left and right panels show the maps obtained without and with the add of the cluster bulk velocity, respectively. The angles $\theta_{\text {los }}$ of the corresponding lines of sight are specified on top of each map. Contours are plotted from $-5 \sigma$ to $5 \sigma$, with dashed(solid) lines for negative(positive) values. The maximum and minimum values in the maps have been set to $\pm 30 \mu \mathrm{K}$ for displaying purposes (see colour version of the figure in the online edition). 


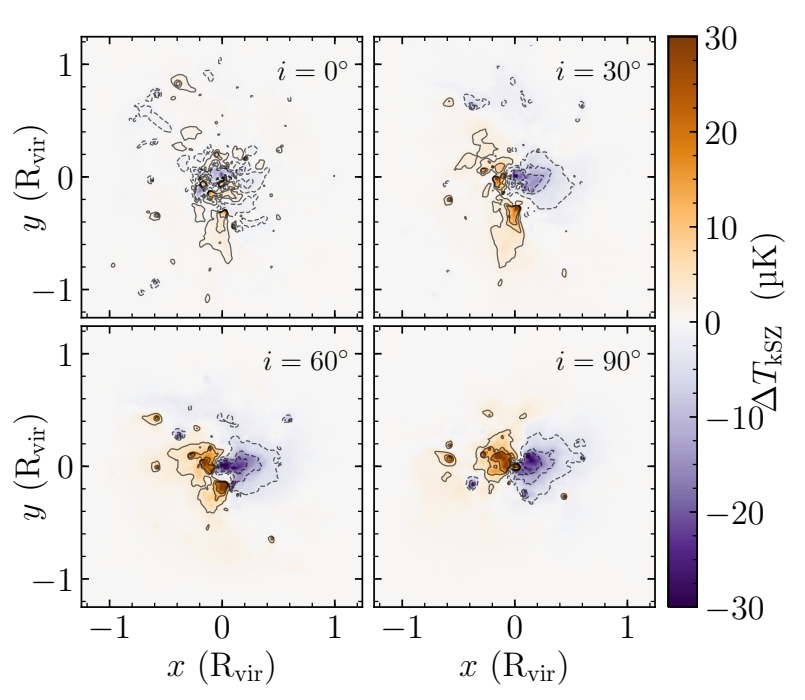

Figure 5. Maps of the kSZ effect of cluster 93, for different values of the angle $i$, without accounting for the contribution of the bulk motion. The dipole pattern weakens from edge-on to face-on with respect to the rotation axis (see colour version of the figure in the online edition).

that, since they extend over scales much smaller than the dipole scale, their presence does not affect dramatically the results from the fit to the theoretical maps of the rotational component of the kSZ. For this reason, the $\Delta T_{\mathrm{kSZ}}$ range in the figures is set to $\pm 30 \mu \mathrm{K}$, in order to fit to the dynamic range of the dipole without being affected by substructure outliers. The other five clusters in the sample show maps with very similar features, as shown in Appendix A.

To highlight the impact of different orientations of the line of sight with respect to the rotation axis, we show in Fig. 5 the maps of cluster 93 at $\theta_{\text {los }}=0^{\circ}$ taken at different angles $i$. We verify that the dipole is clearly visible in the case of orthogonal line of sight with respect to the rotation axis, with decreasing amplitude for decreasing values of $i$, consistently with the expectations. Unfortunately, the contribution from the $\sin i$ term and the $v_{t 0}$ parameter cannot be discriminated in the observed signal. For this reason we set $i=90^{\circ}$ in the rest of the analysis.

\section{RESULTS AND DISCUSSION}

In order to recover the rotational properties of our test clusters, we use the Levenberg-Marquardt least-square algorithm (Moré 1978) to compute a pixel-to-pixel fit to the synthetic kSZ maps described in section 3.2. We treat separately the purely rotational case, referring to the theoretical model given by equation (2), and the full case accounting also for the cluster bulk motion, referring to the model of equation (3). The six parameters of the radial profile of the electron number density are kept fixed, with the values listed in Table 2 of section 3.1. The free parameters we recover from the fit to the $\mathrm{kSZ}$ maps are the scale radius, $r_{0}$, and the scale velocity, $v_{t 0}$, introduced in equation (6). In the full case including the bulk motion, we treat the cluster bulk velocity projected on the line of sight $\left(v_{\text {bulk }}\right)$ as an additional free parameter. In order to account for a possi- ble non-horizontal alignment of the dipole with respect to the projection of the rotation axis ( $y$ axis in the maps), we repeat the fit also adding another free parameter to the theoretical model of the kSZ map. It consists in an offset, $\delta \phi$, added to the azimuthal angle $\phi$, which partially accounts for the error in the estimate of the correct orientation of the rotation axis of the ICM. If $\delta \phi=0^{\circ}$, it is correct to assume the direction of the angular momentum of the gas at virial radius as the rotation axis. We verify that this offset takes values smaller than $10^{\circ}$ in most cases, and that it does not affect significantly the results from the fit. For this reason, we refer only to the case with $\delta \phi=0^{\circ}$ in all the forthcoming results.

Fig. 6 shows the kSZ maps generated from the data with the corresponding best-fit theoretical maps, all smoothed at 20 arcsec, without (left panel) and with (right panel) the bulk motion, in the case of cluster 93. Similar maps for the complete sample showing the best lines of sight are reported in Appendix A. We also show in Fig. 7 - for cluster 93 as before - the central cuts through the same maps of Fig. 6 , to better highlight how our procedure recovers the features of the signal. It can be seen that, in general, the theoretical model is appropriate to describe the data in both cases of subtraction and adding of the cluster bulk velocity. The signal in the data maps may be larger of a factor of $\approx 3$ at most with respect to the best-fit maps in some regions, because of small-scale outliers due to sub-structures (that can be clearly spotted in the plots of Fig. 7), whose contribution generally changes from one line of sight to another (see also Fig. 4). Table 3 lists the $r_{0}$ and $v_{t 0}$ parameters estimated from the fit. Since they should have consistent values independently on the observed projection, we report the average and standard deviation over the different lines of sight we considered. We find that the values of both parameters are in agreement within one standard deviation when comparing the two cases of subtraction and add of the bulk term (listed in the left and centre columns of Table 3, respectively). This indicates that, in principle, this procedure is able to disentangle the signal produced by rotation from the one given by the bulk motion. The comparison between these results and the values derived from the fit to the velocity inferred directly from the simulation data (listed in the right columns in Table 3), shows that the scale radius $r_{0}$ is consistent within one standard deviation. The values of the $v_{t 0}$ parameter are overestimated with respect to the expected ones by a factor of $\sim 1.5(1.6)$ on average, when subtracting(adding) the bulk motion. Indeed, they reach fractions larger than 80 per cent of the circular velocity at the virial radius (which is $\gtrsim 1000 \mathrm{~km} \mathrm{~s}^{-1}$ for all the clusters). Nevertheless, there is agreement within one standard deviation for almost all clusters. A noticeable exception is given by cluster 98 , for which we get a larger overestimate (of factors 3.1 and 2.8 without and with the cluster bulk, respectively), and significantly larger errors. Such discrepancies may be due, in general, to a less efficient reconstruction of the dipole because of a higher impact from irregularities in the gas density distribution, and from high-velocity sub-structures, especially in the outer regions (see e.g. the corresponding panels in Fig. A1). In order to give a quantitative indication of the kSZ signal coming from rotation, we measure the amplitude of the dipole from the best-fit theoretical maps in the rotation-only case. Values are listed in Table 4, as derived 

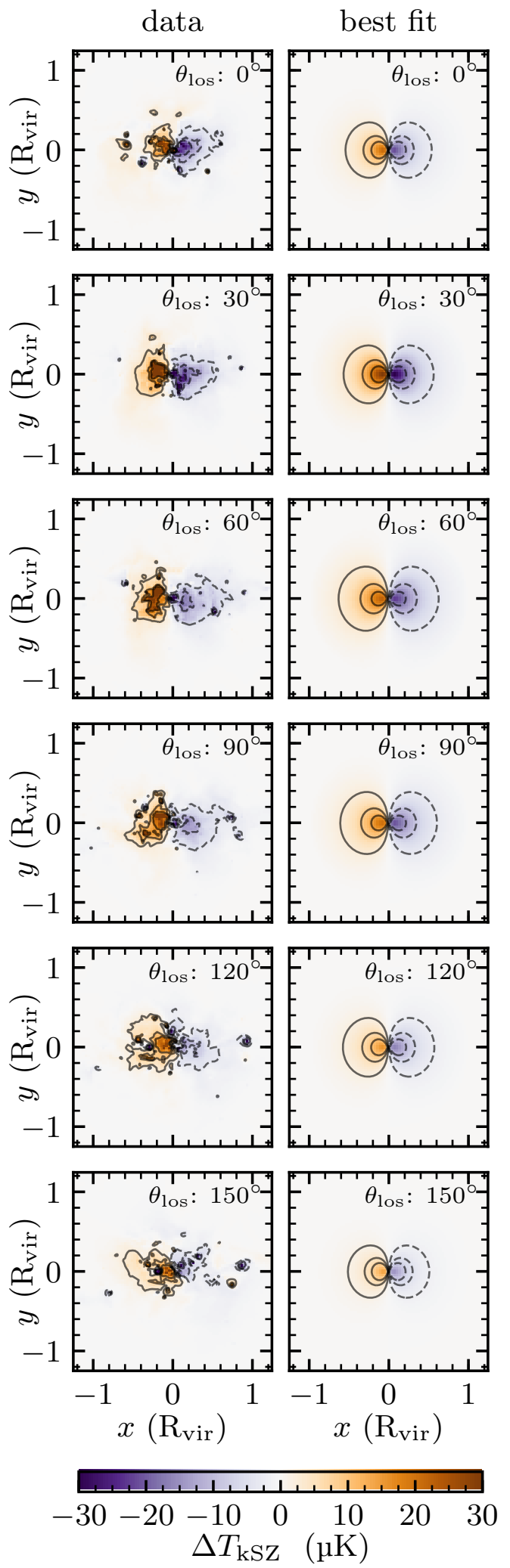

(a) without bulk motion

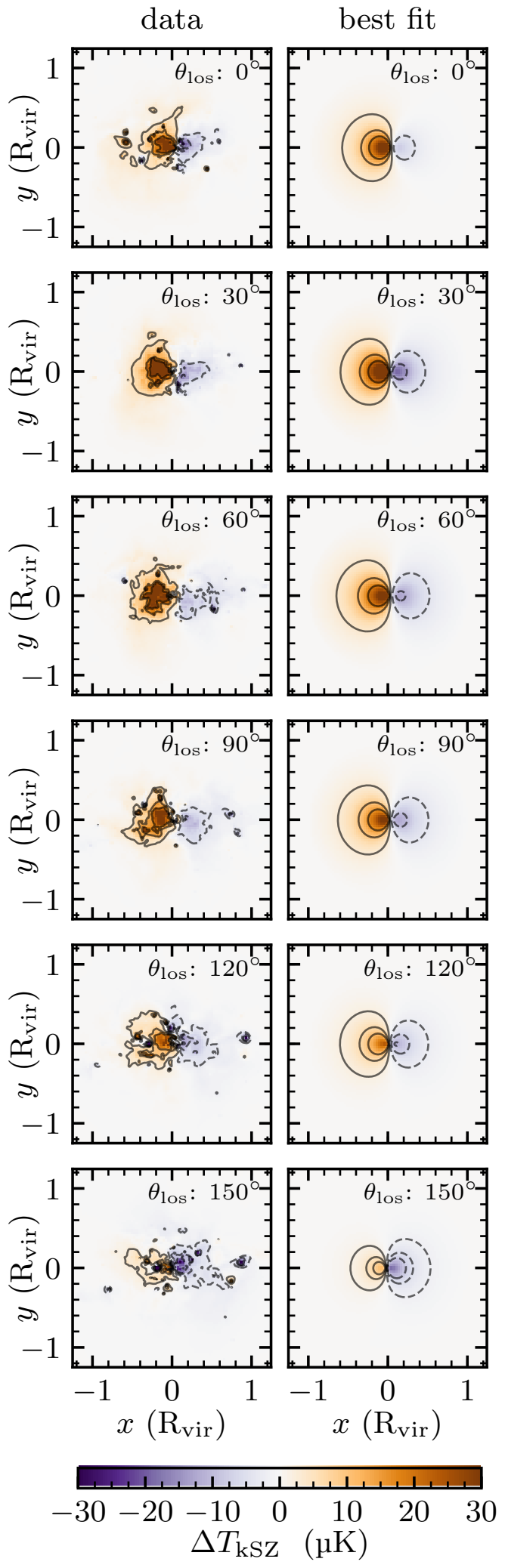

(b) with bulk motion

Figure 6. Comparison between the kSZ temperature maps of cluster 93 and the corresponding best-fit maps, without and with accounting for the cluster bulk velocity (left and right panels, respectively). Contours are plotted from $-5 \sigma$ to $5 \sigma$, with dashed(solid) lines for negative(positive) values. The colorbar is set to $\pm 30 \mu \mathrm{K}$ for displaying purposes (see colour version of the figure in the online edition). 
Table 3. Best-fit values of the free parameters $r_{0}$ (scale radius) and $v_{t 0}$ (scale velocity) of equation (6). Left and middle columns report the average values with their standard deviation computed over all the lines of sight, as derived from the fit to the kSZ maps without and with the bulk term, respectively. Right columns lists the expected values for the parameters, derived from the fit to the tangential velocity data, $v_{t}$, extracted from the simulation. Velocities are given in units of the circular velocity at the virial radius, $v_{\mathrm{circ}}=\sqrt{G M_{\mathrm{vir}} / R_{\mathrm{vir}}}$.

\begin{tabular}{ccccccc}
\hline \multirow{2}{*}{ cluster ID } & \multicolumn{2}{c}{ fit to kSZ without bulk } & \multicolumn{2}{c}{ fit to kSZ with bulk } & \multicolumn{2}{c}{ fit to $v_{t}$ data } \\
\cline { 2 - 7 } & $r_{0}\left(R_{\text {vir }}\right)$ & $v_{t 0}\left(v_{\text {circ }}\right)$ & $r_{0}\left(R_{\text {vir }}\right)$ & $v_{t 0}\left(v_{\text {circ }}\right)$ & $r_{0}\left(R_{\text {vir }}\right)$ & $v_{t 0}\left(v_{\text {circ }}\right)$ \\
\hline 46 & $0.34 \pm 0.09$ & $0.84 \pm 0.04$ & $0.37 \pm 0.05$ & $0.95 \pm 0.17$ & $0.36 \pm 0.22$ & $0.56 \pm 0.17$ \\
93 & $0.34 \pm 0.08$ & $0.87 \pm 0.27$ & $0.34 \pm 0.07$ & $0.88 \pm 0.28$ & $0.49 \pm 0.27$ & $0.58 \pm 0.20$ \\
98 & $0.38 \pm 0.30$ & $1.53 \pm 0.67$ & $0.33 \pm 0.25$ & $1.39 \pm 0.65$ & $0.57 \pm 0.62$ & $0.49 \pm 0.29$ \\
103 & $0.29 \pm 0.13$ & $0.82 \pm 0.09$ & $0.28 \pm 0.11$ & $0.83 \pm 0.09$ & $0.47 \pm 0.35$ & $0.52 \pm 0.19$ \\
205 & $0.20 \pm 0.08$ & $0.96 \pm 0.08$ & $0.24 \pm 0.09$ & $0.86 \pm 0.19$ & $0.27 \pm 0.14$ & $0.65 \pm 0.17$ \\
256 & $0.32 \pm 0.12$ & $1.00 \pm 0.23$ & $0.33 \pm 0.11$ & $1.02 \pm 0.24$ & $0.37 \pm 0.20$ & $0.61 \pm 0.22$ \\
\hline
\end{tabular}

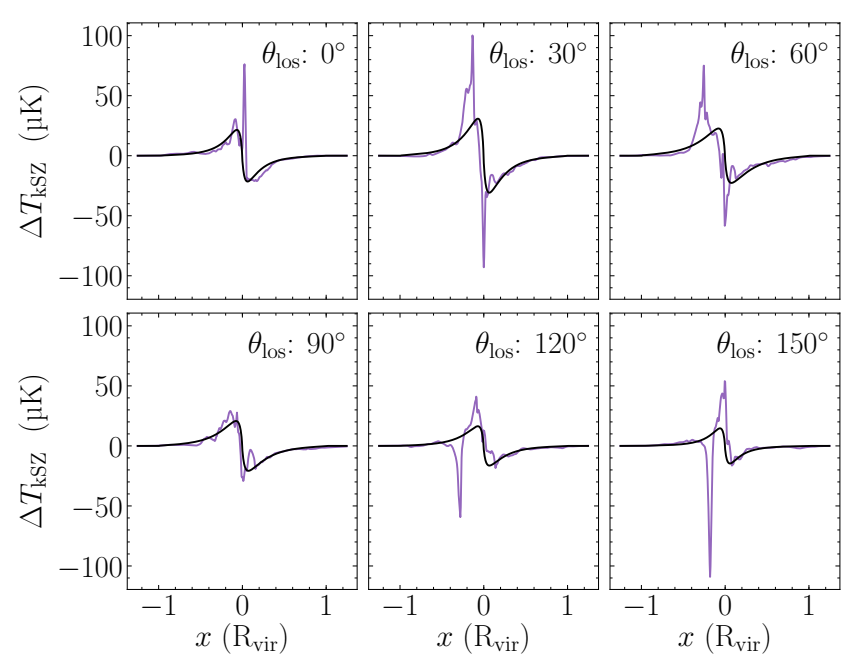

(a) without bulk motion

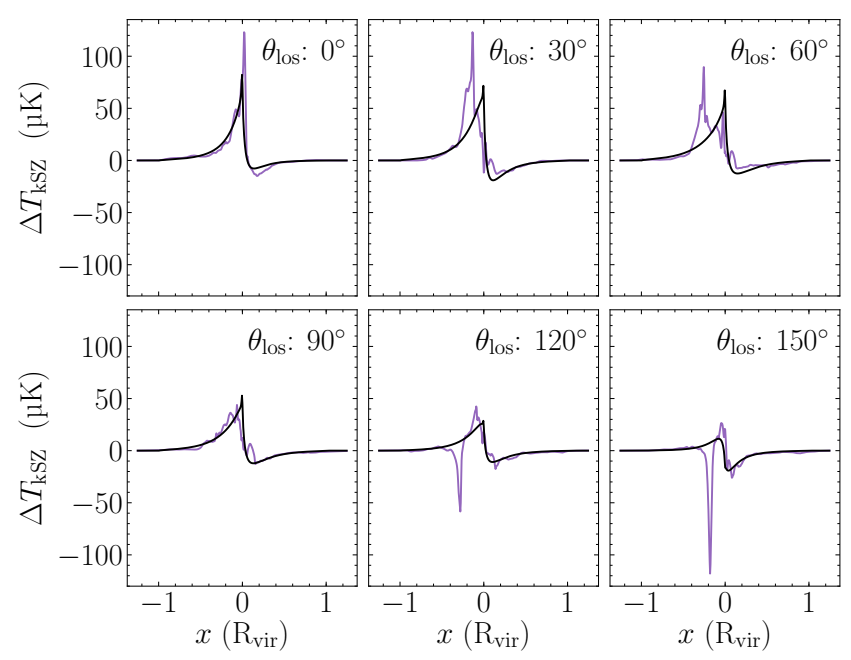

(b) with bulk motion

Figure 7. Central cuts from the kSZ maps of cluster 93, without (top panel) and with (bottom panel) the cluster bulk velocity, for different lines of sight. Purple curves represent the cut through the data maps, while black curves represent the cut through the best-fit maps. The presence of high-velocity particles can be seen as outliers at small scales.
Table 4. Amplitude of the kSZ temperature signal measured from the best-fit maps. The $A_{\text {dip }}$ column refers to the amplitude of the dipole averaged over all the lines of sight, as derived from the fit to the maps without the bulk motion. $A_{\text {bulk }}$ refers instead to the maximum amplitude of the best fit to the maps accounting for the cluster bulk motion.

\begin{tabular}{ccc}
\hline cluster ID & $A_{\text {dip }}(\mu \mathrm{K})$ & $A_{\text {bulk }}(\mu \mathrm{K})$ \\
\hline 46 & $10.8 \pm 2.5$ & -57.5 \\
93 & $21.1 \pm 5.2$ & 82.1 \\
98 & $24.4 \pm 9.2$ & -77.9 \\
103 & $16.5 \pm 2.9$ & -99.4 \\
205 & $20.9 \pm 5.1$ & 68.8 \\
256 & $24.4 \pm 4.3$ & -143.1 \\
\hline
\end{tabular}

Table 5. Normalized difference between the recovered $v_{\text {bulk }}$ and the true one estimated from the simulation, $v_{\text {bulk,sim. }}$. Values along the columns refer to the six different lines of sight.

\begin{tabular}{ccccccc}
\hline \multirow{2}{*}{ cluster ID } & \multicolumn{5}{c}{$v_{\text {bulk }} / v_{\text {bulk,sim }}-1$} \\
\cline { 2 - 6 } & $0^{\circ}$ & $30^{\circ}$ & $60^{\circ}$ & $90^{\circ}$ & $120^{\circ}$ & $150^{\circ}$ \\
\hline 46 & -0.04 & -0.14 & -0.22 & -0.32 & -0.68 & -0.11 \\
93 & -0.01 & 0.28 & 0.24 & 0.04 & -3.39 & -0.68 \\
98 & 0.77 & -0.21 & 0.70 & -4.96 & -0.20 & -0.84 \\
103 & -0.04 & -0.17 & -0.43 & -0.38 & -0.04 & 0.01 \\
205 & 0.13 & -0.92 & -0.09 & -0.01 & -0.03 & -0.11 \\
256 & -0.10 & -0.17 & -0.28 & -0.30 & -0.61 & 0.14 \\
\hline
\end{tabular}

from the average over all the explored projections. It can be seen that the dispersion across different lines of sight is of the order of 38 per cent at most, while average values are of the order of few tens of $\mu \mathrm{K}$. This result is in agreement with the predictions by Chluba \& Mannheim (2002), that indicates dipole amplitudes ranging between $\sim 4$ and $\sim 30 \mu \mathrm{K}$ (assuming a solid body rotation). Table 4 also reports the maximum amplitude, $A_{\text {bulk }}$, measured in the best-fit maps accounting for the cluster bulk motion. We verify that, as expected, this quantity is linearly proportional to the projected bulk velocity, $v_{\text {bulk. }}$. Using these values it is possible to compute the ratio $A_{\text {dip }} / A_{\text {bulk }}$, in order to estimate how much does the rotation contribute to the total $\mathrm{kSZ}$ signal in the case in which the projection of the cluster bulk velocity takes its maximum value. The average value of this ratio is $\sim 0.23$, confirming that very high sensitivities are needed to measure the effect of a rotation, provided the best observational conditions. The recovered values of $v_{\text {bulk }}$ are of 


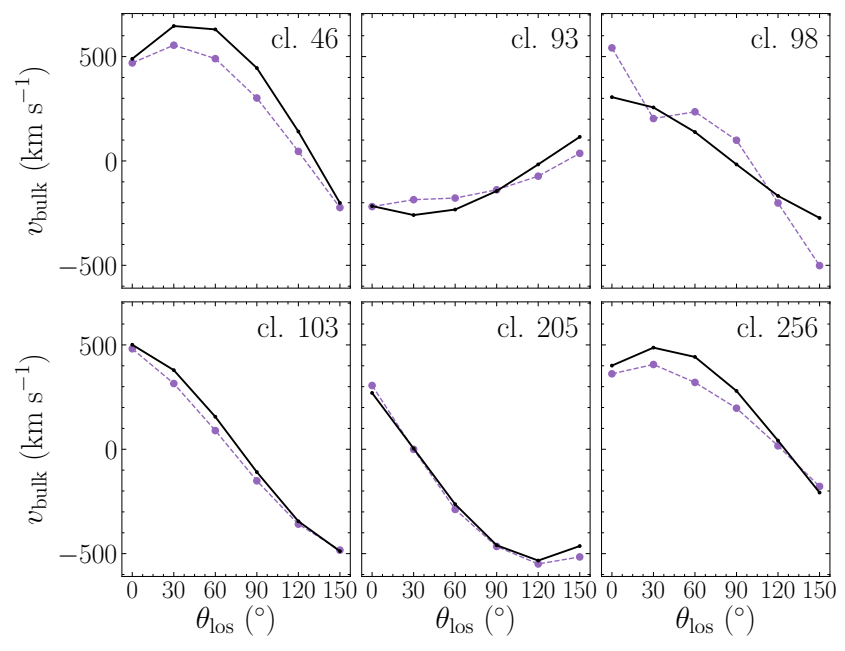

Figure 8. Values of the projected cluster bulk velocity, $v_{\text {bulk }}$, as a function of the angle $\theta_{\text {los }}$ identifying the different lines of sight. Purple dots with dashed line represent the estimate from the fit to the maps with the bulk term. Black dots with solid line are the true values extracted from the simulation.

the order of hundreds of $\mathrm{kms}^{-1}$, and they are fairly compatible with the true values from the simulation. We show their comparison in Fig. 8, where it can also be seen the sinusoidal behaviour of the different projections with varying $\theta_{\text {los }}$. In Table 5 we report the differences between $v_{\text {bulk }}$ recovered from the fit to the $\mathrm{kSZ}$ maps and the true value from the simulation, $v_{\text {bulk,sim }}$, normalized to $v_{\text {bulk,sim }}$ itself. Values are generally underestimated by few tens of per cent at most projections; differences are more significant for lines of sight in which the projected bulk velocity takes absolute values smaller than $100 \mathrm{~km} \mathrm{~s}^{-1}$. The possibility of recovering the bulk velocity term with this procedure is an important result of this work. Indeed, the use of the rotational kSZ effect with complementary observables, e.g. higher order corrections terms to the Kompaneets approximation, or the degree of CMB polarization induced by the $\mathrm{kSZ}$, could give an estimate of the three-dimensional cluster velocity (Birkinshaw 1999).

The simplifying assumptions we have made, e.g. the orthogonal orientation of the los with respect to the rotation axis, and the poor error constraints we get in the final estimates of the parameters could be limiting factors for this analysis. Nevertheless, this study is intended to quantify the amount of kSZ signal that would be produced by ICM rotation at the best observational conditions, also to get a possible validation of the vp $2 \mathrm{~b}$ model. Some enhancements to get more robust results can include e.g. a proper modelling of the sub-structures to be included in the theoretical map for a more accurate fit. Also, a deeper approach focused on a more quantitative assessment of the feasibility of such challenging observations at millimetre wavelengths is ongoing, by accounting for the full instrumental effects and contamination from astrophysical sources and SZ background.

\section{SUMMARY AND CONCLUSIONS}

In this paper we address the study of the rotation of the ICM in simulated galaxy clusters through the maps of the $\mathrm{kSZ}$ effect. In this preliminary analysis, we select a sample of six particularly relaxed and possibly rotating objects from MUSIC simulations studied in B17, and we fit their kSZ temperature maps to a theoretical model based on the one proposed by CC02. We adopt a simplified Vikhlinin model for the electron number density, and the vp2b model of B17 for the angular velocity. We study both a simplified case which does not account for the cluster bulk motion, in order to get only the rotational signal, and the complete case that includes an additional term depending on the cluster bulk velocity projected on the line of sight. We explore six different lines of sight for both cases, picking them all orthogonal to the rotation axis, in order to maximise the amplitude of rotational signal. The main results from the fit to our maps can be summarized as follows:

- with our procedure we can recover the parameters of the radial profile of the rotational velocity within one standard deviation in the case of the scale radius, and within two standard deviations at most in the case of the scale velocity, by averaging over all the lines of sight. These results are poorly affected by the small-scale outliers produced by high-velocity sub-structures located within the cluster virial radius, at most projections;

- the amplitude of the best-fit dipole is consistent with the estimates found in the literature for relaxed systems. From a comparison between the amplitude derived without and with the bulk motion, we estimate that the rotational contribution to the total $\mathrm{kSZ}$ signal is, on average, of the order of 23 per cent in the best observational conditions;

- the projection of the bulk velocity on the line of sight estimated from the full $\mathrm{kSZ}$ model shows differences of few per cent at most projections with respect to the true values from the simulation. As expected, it has a sinusoidal behaviour as a function of the angle identifying each particular line of sight.

We plan to refine the analysis presented in this work by accounting for the full pipeline of NIKA2, including instrumental and astrophysical contaminants, to possibly apply this study to observations of real clusters. This work is part of a larger project on the feasibility of the characterization of ICM rotation, that will include the complementary analysis of multi-frequency data generated from MUSIC clusters. In particular, we are working on the analysis of the dynamics of galaxy members in our cluster sample, to investigate the possible co-rotation between baryonic components. In this context, the future Euclid mission (Amiaux et al. 2012) could open new interesting possibilities for observations in the optical band. We also plan to account for X-ray data, given their complementarity to the SZ effect to derive cluster properties and dynamics. For instance, the upcoming X-ray satellite Athena (Barcons et al. 2015) will feature an unprecedented sensitivity and resolution for the characterization of spectral lines, that would turn to be extremely useful to constrain the ICM kinetic properties. Lastly, given the recent developments of the pymsz code, another possible future development of the present work applied to synthetic clusters would include the treatment of the full signal from 
tSZ and kSZ, to recover e.g. cluster properties and their impact on cosmology.

\section{ACKNOWLEDGEMENTS}

This work has been supported by funding from Sapienza University of Rome - Progetti di Ricerca Medi 2017, prot. RM11715C81C4AD67. ASB acknowledges founding from Sapienza University of Rome - Progetti per Avvio alla Ricerca Anno 2017, prot. AR11715C82402BC7. WC and GY are supported by the Ministerio de Economía y Competitividad and the Fondo Europeo de Desarrollo Regional (MINECO/FEDER, UE) in Spain through grant AYA201563810-P. The authors thank P. Mazzotta for comments and suggestions, and acknowledge G. Cialone for useful discussions. The MUSIC simulations were produced with the Marenostrum supercomputer at the Barcelona Supercomputing Centre thanks to time awarded by Red Española de Supercomputación. This work makes an extensive use of python, particularly of the scipy and matplotlib libraries. 


\section{REFERENCES}

Adam R., et al., 2017, A\&A, 598, A115

Amiaux J., et al., 2012, in Proc. SPIE.

Baldi A. S., et al., 2017, MNRAS, 465, 2584

Barcons X., et al., 2015, JPhCS, 610, 012008

Benson B. A., et al., 2003, ApJ, 592, 674

Bianconi M., Ettori S., Nipoti C., 2013, MNRAS, 434, 1565

Biffi V., Dolag K., Böhringer H., 2011, MNRAS, 413, 573

Biffi V., Dolag K., Böhringer H., 2013, MNRAS, 428, 1395

Biffi V., et al., 2014, MNRAS, 439, 588

Biffi V., et al., 2016, ApJ, 827, 112

Birkinshaw M., 1999, PhR, 310, 97

Chluba J., Mannheim K., 2002, A\&A, 396, 419

Cialone G., et al., 2018, MNRAS, 477, 139

Cooray A., Chen X., 2002, ApJ, 573, 43

Cui W., et al., 2016, MNRAS, 458, 4052

Cui W., et al., 2017, MNRAS, 464, 2502

Dupke R. A., Bregman J. N., 2002, ApJ, 575, 634

Eckert D., et al., 2018, preprint (arXiv:1805.00034)

Fang T., Humphrey P., Buote D., 2009, ApJ, 691, 1648

Fixsen D. J., 2009, ApJ, 707, 916

Gottlöber S., Yepes G., 2007, ApJ, 664, 117

Hamden E. T., et al., 2010, ApJ, 716, L205

Hand N., et al., 2012, Phys. Rev. Lett., 109, 041101

Hitomi Collaboration et al., 2016, Nature, 535, 117

Hitomi Collaboration et al., 2018, PASJ, 70, 9

Holzapfel W. L., et al., 1997, ApJ, 481, 35

Hwang H. S., Lee M. G., 2007, ApJ, 662, 236

Kashlinsky A., et al., 2008, ApJ, 686, L9

Kashlinsky A., et al., 2009, ApJ, 691, 1479

Komatsu E., et al., 2011, ApJS, 192, 18

Kompaneets A. S., 1957, JETP, 4, 730

Lau E. T., Kravtsov A. V., Nagai D., 2009, ApJ, 705, 1129

Li Y.-C., et al., 2018, Phys. Rev. D, 97, 23514

Liu A., et al., 2015, ApJ, 809, 27

Manolopoulou M., Plionis M., 2017, MNRAS, 465, 2616

Mather J. C., et al., 1999, ApJ, 512, 511

Meneghetti M., et al., 2010, A\&A, 514

Moré J. J., 1978, in Lecture Notes in Mathematics, Vol. 630, Numerical Analysis. Springer Berlin Heidelberg, pp 105-116

Nagai D., Kravtsov A. V., Kosowsky A., 2003, ApJ, 587, 524

Nelson K., et al., 2012, ApJ, 751, 121

Planck Collaboration et al., 2016, A\&A, 586, A140

Prada F., et al., 2012, MNRAS, 423, 3018

Rasia E., Tormen G., Moscardini L., 2004, MNRAS, 351, 237

Rasia E., et al., 2006, MNRAS, 369, 2013

Rephaeli Y., 1995, ARA\&A, 33, 541

Ricker P. M., Sarazin C. L., 2001, ApJ, 561, 621

Sayers J., et al., 2013, ApJ, 778, 52

Sayers J., et al., 2016, ApJ, 820, 101

Sembolini F., et al., 2013, MNRAS, 429, 323

Sembolini F., et al., 2014, MNRAS, 440, 3520

Springel V., 2005, MNRAS, 364, 1105

Sunyaev R. A., Zel'dovich Y. B., 1970, Ap\&SS, 7, 20

Sunyaev R. A., Zel'dovich Y. B., 1980, ARA\&A, 18, 537

Sunyaev R. A., Norman M. L., Bryan G. L., 2003, AstL, 29, 783

Tchernin C., et al., 2016, A\&A, 595, A42

Tovmassian H. M., 2015, Ap, 58, 328

Vikhlinin A., et al., 2006, ApJ, 640, 691

Voit G. M., 2005, RvMP, 77, 207

Zhang C., Yu Q., Lu Y., 2018, ApJ, 855, 36

\section{APPENDIX A: MAPS FOR THE WHOLE SAMPLE}

We show in this section the $\mathrm{kSZ}$ temperature maps of all the six clusters in the sample. The data maps, computed as detailed in section 3 and smoothed at 20 arcsec, are shown in Fig. A1 without the bulk motion. Fig. A2 shows the same maps without the subtraction of the bulk velocity. The best fit maps together with the data maps are shown only for the best lines of sight in Fig. A3, without (top panels) and with (bottom panels) the bulk motion.

This paper has been typeset from a $\mathrm{T}_{\mathrm{E}} \mathrm{X} / \mathrm{AT}_{\mathrm{E}} \mathrm{X}$ file prepared by the author. 


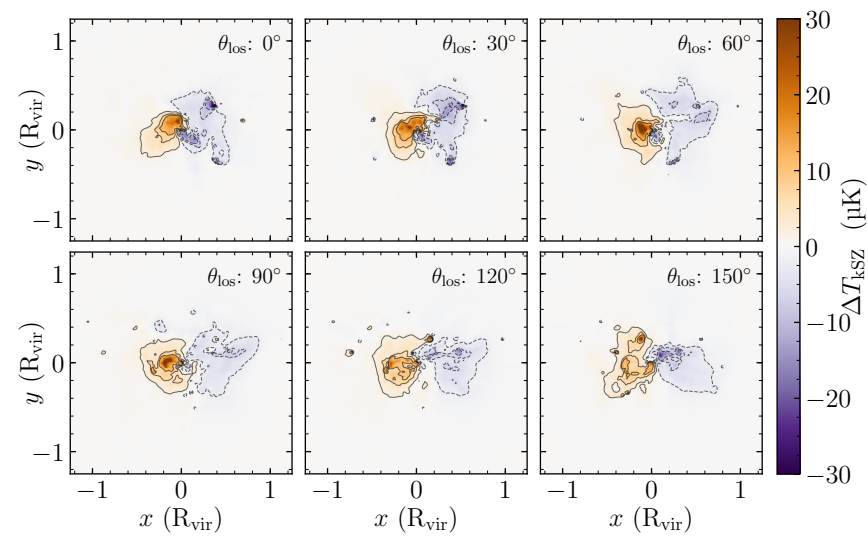

(a) cl. 46

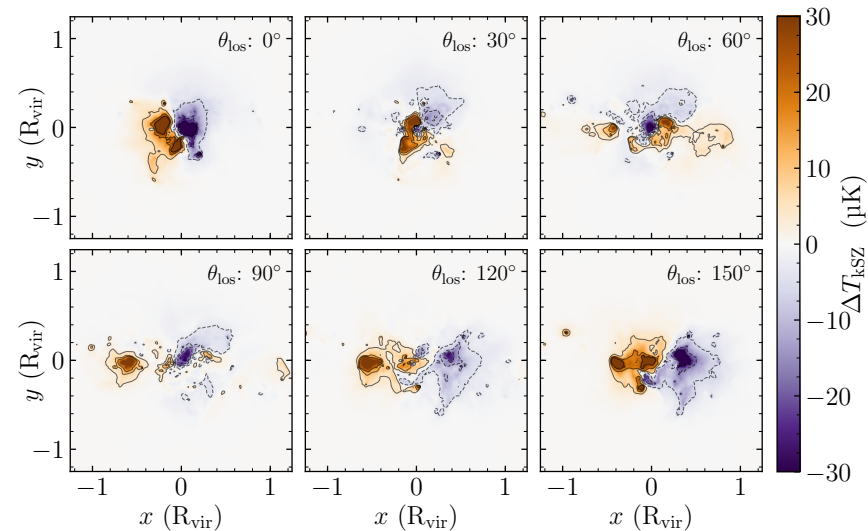

(c) cl. 98

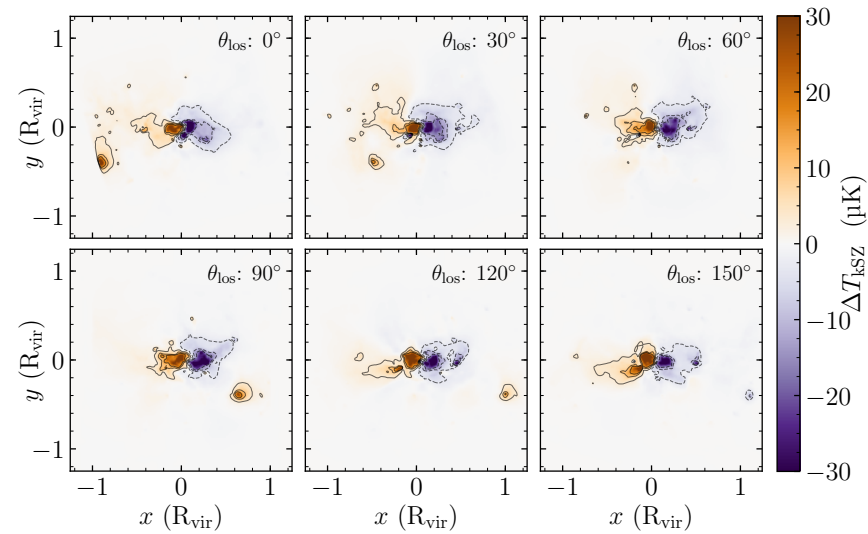

(e) cl. 205

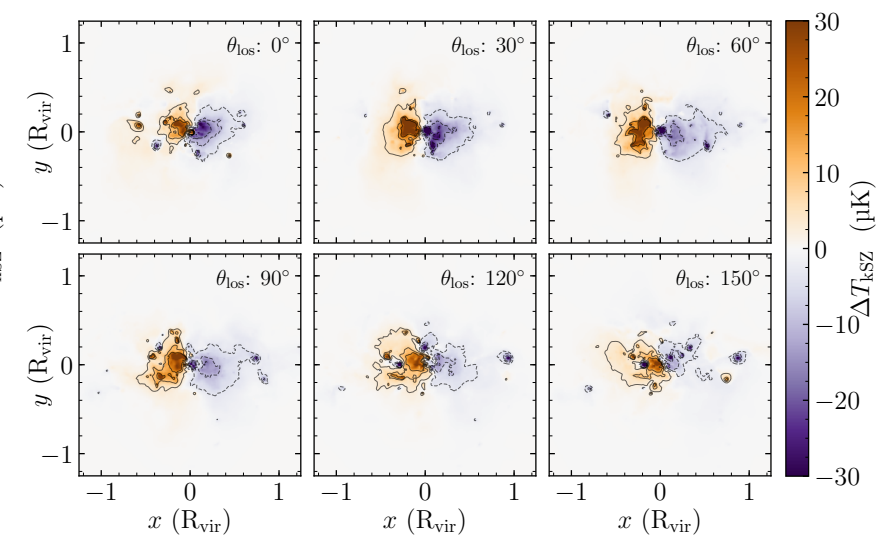

(b) cl. 93

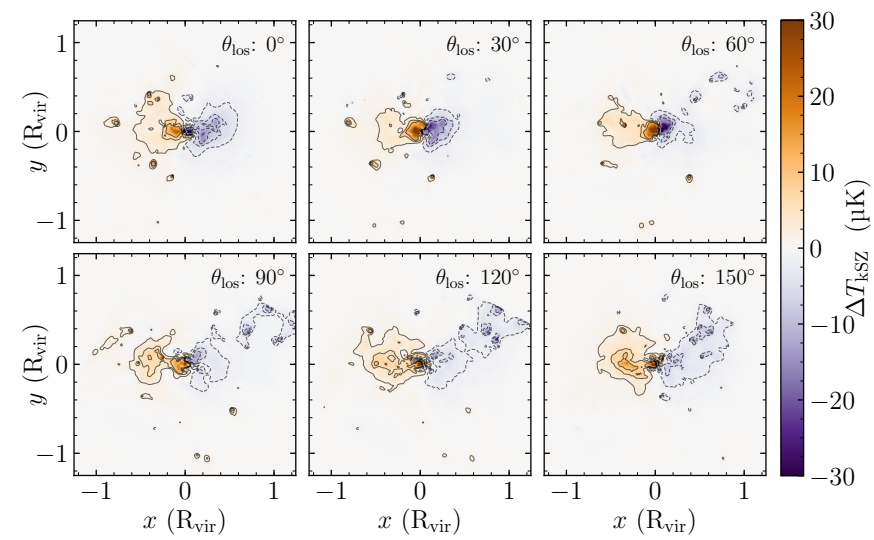

(d) cl. 103

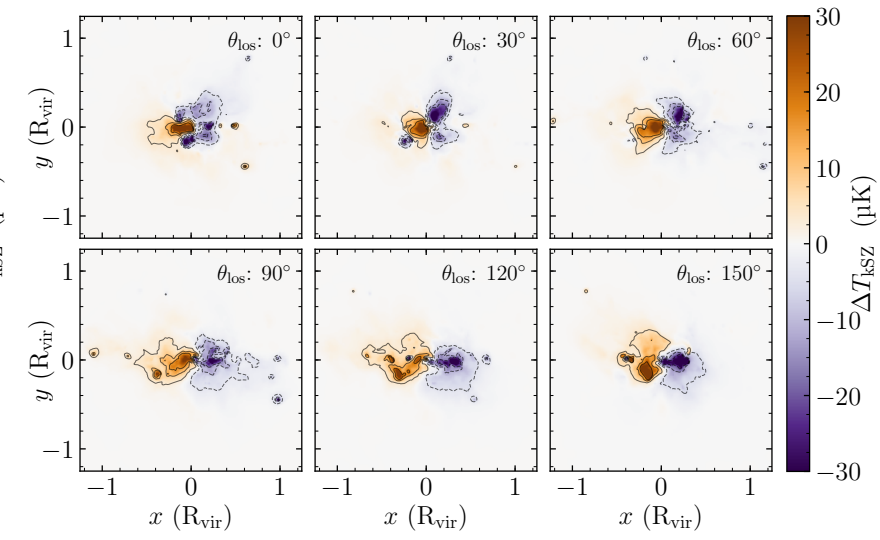

(f) cl. 256

Figure A1. Maps of the temperature shift produced by the kSZ effect for all the clusters in the sample, obtained from different projections as described in the text, and smoothed at 20 arcsec. The angles of the corresponding lines of sight, taken on the plane orthogonal to the rotation axis, are specified on top of each map. Contours are plotted from $-5 \sigma$ to $5 \sigma$, with dashed(solid) lines for negative(positive) values. The ranging values in the map have been set to $\pm 30 \mu \mathrm{K}$ for displaying purposes (see colour version of the figure in the online edition). 


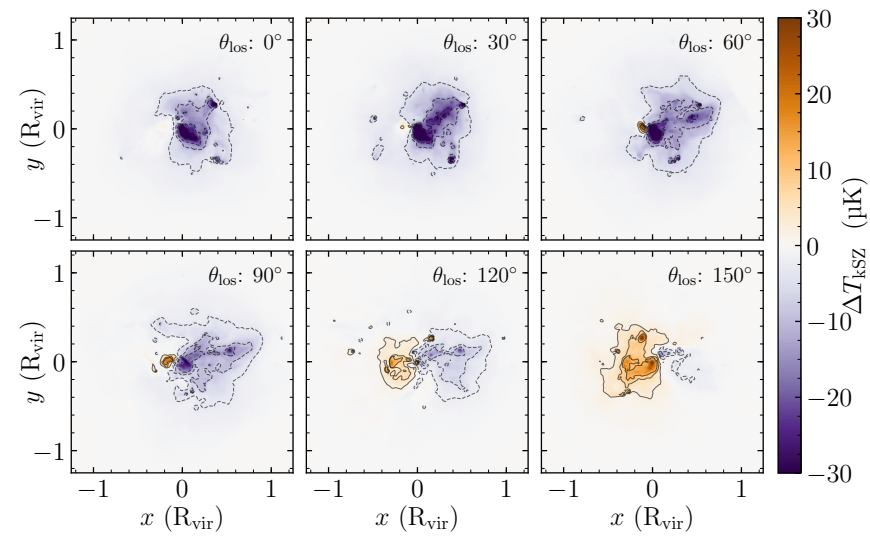

(a) cl. 46

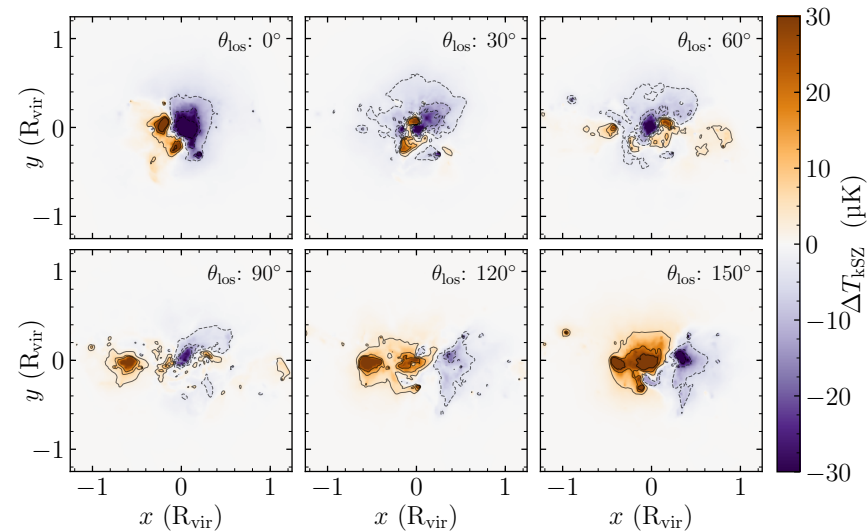

(c) cl. 98

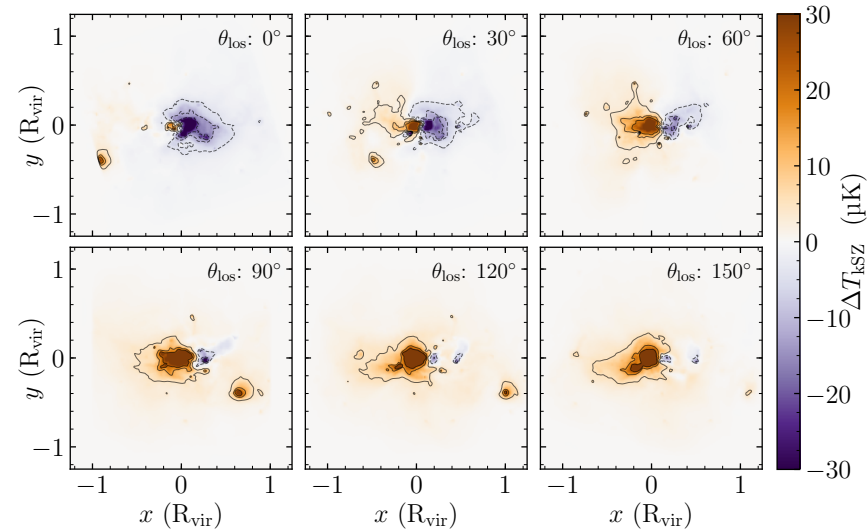

(e) cl. 205

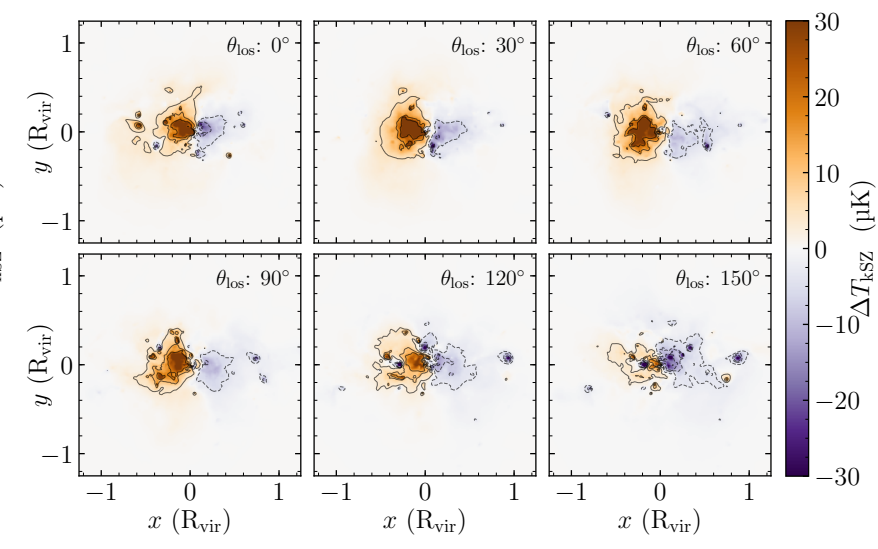

(b) cl. 93

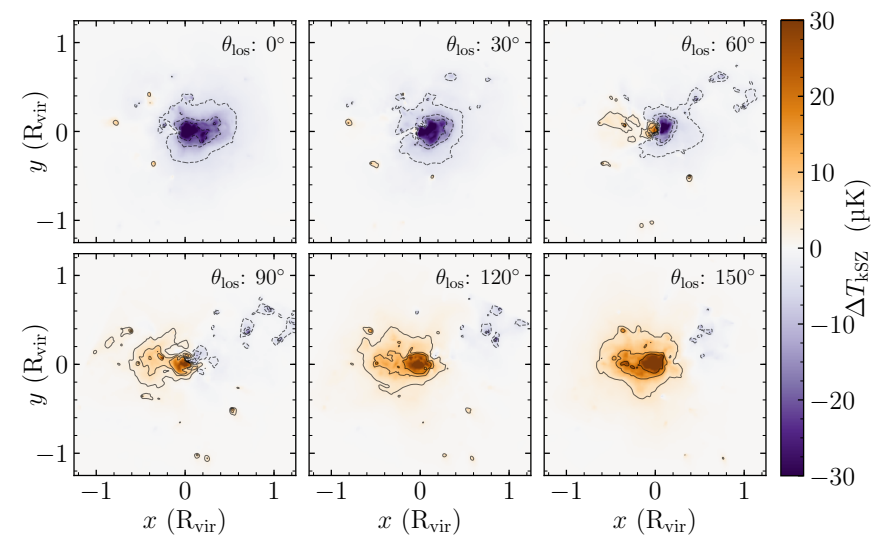

(d) cl. 103

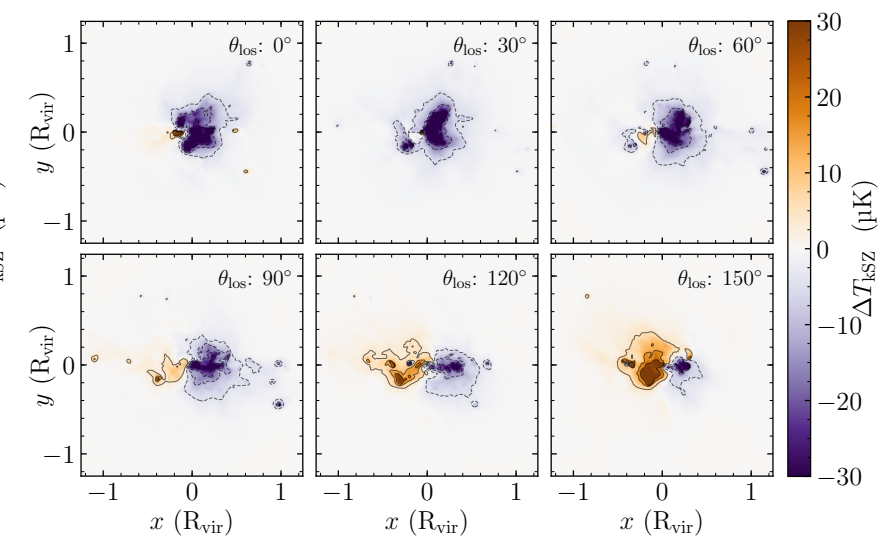

(f) cl. 256

Figure A2. Maps of the temperature shift produced by the kSZ effect accounting for the bulk motion for all the clusters in the sample, obtained from different projections as described in the text, and smoothed at 20 arcsec. The angles of the corresponding lines of sight, taken on the plane orthogonal to the rotation axis, are specified on top of each map. Contours are plotted from $-5 \sigma$ to $5 \sigma$, with dashed(solid) lines for negative(positive) values. The ranging values in the map have been set to $\pm 30 \mu \mathrm{K}$ for displaying purposes (see colour version of the figure in the online edition). 


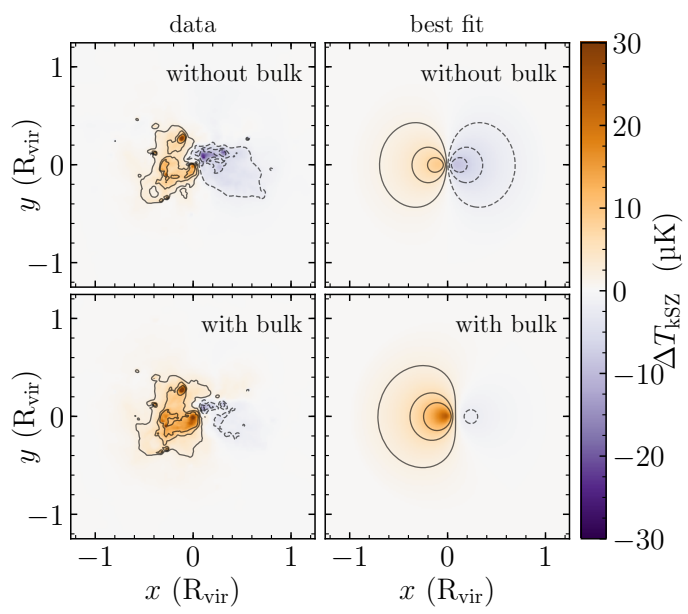

(a) cl. 46

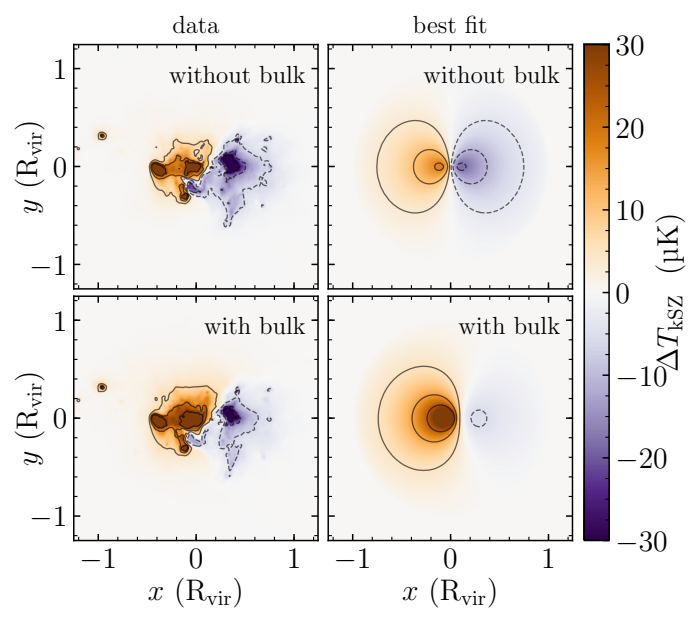

(c) cl. 98

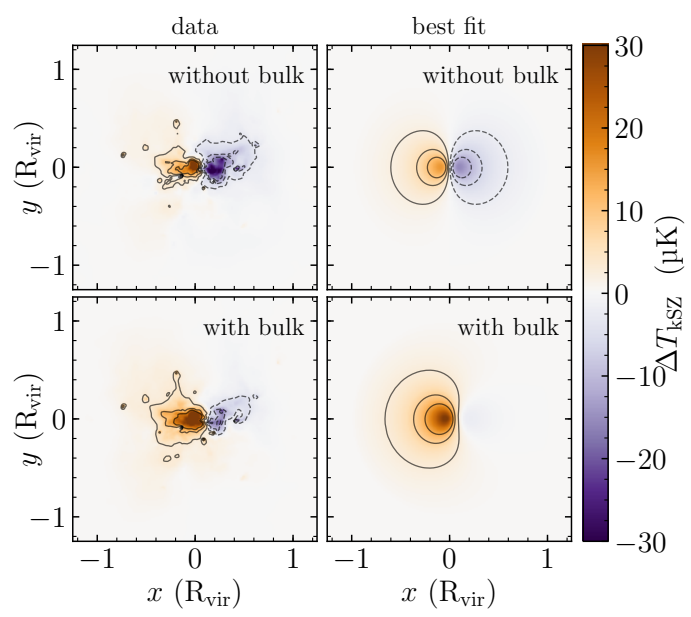

(e) cl. 205

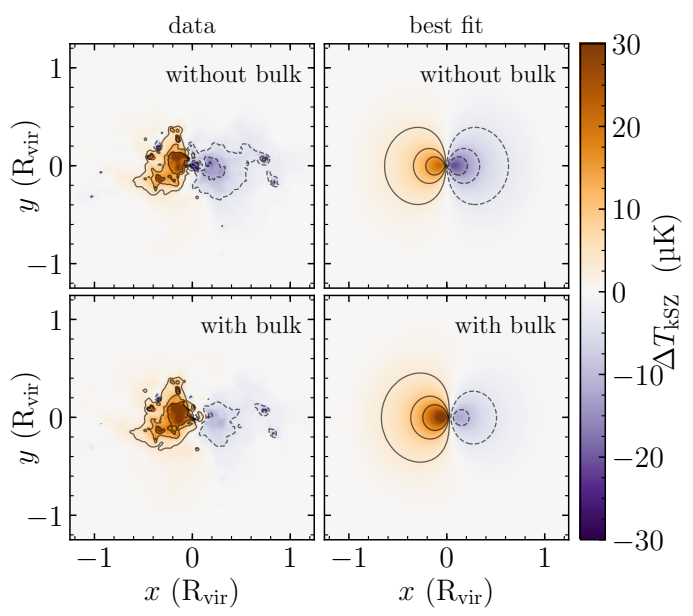

(b) cl. 93

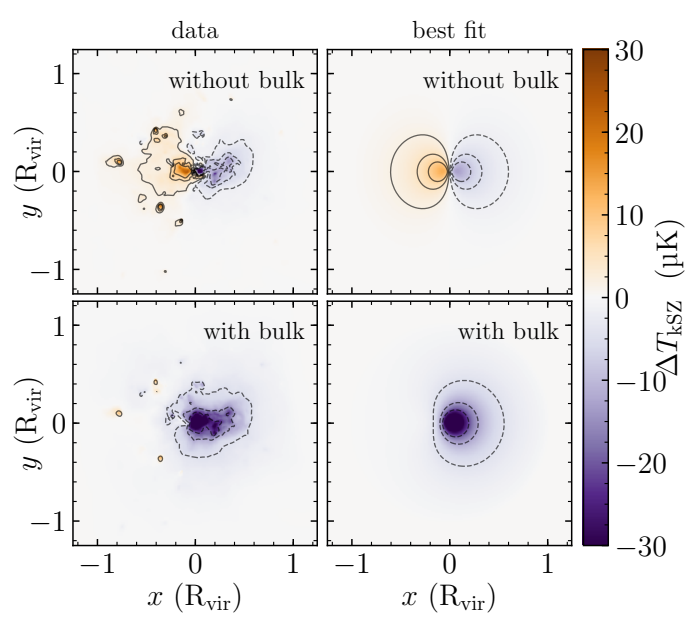

(d) cl. 103

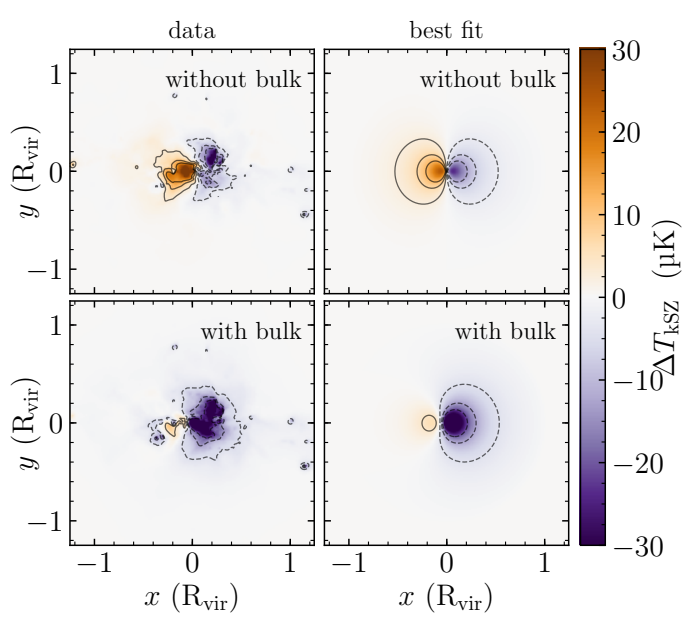

(f) cl. 256

Figure A3. Maps of the temperature shift produced by the kSZ effect at the best projection for all the clusters in the sample smoothed at 20 arcsec, with the corresponding best fit. Maps in the top and bottom panels refer to the case without and with the bulk velocity, respectively. Contours are plotted from $-5 \sigma$ to $5 \sigma$, with dashed(solid) lines for negative(positive) values. The ranging values in the map have been set to $\pm 30 \mu \mathrm{K}$ for displaying purposes (see colour version of the figure in the online edition). 